\title{
BOSQUES SECUNDARIOS DESARROLLADOS EN TIERRAS AGRÍCOLAS ABANDONADAS EN LA REGIÓN DEL PACÍFICO DE NICARAGUA. PROCEDIMIENTO PARA LA RESTAURACIÓN DE ECOSISTEMAS FORESTALES DEGRADADOS
}

\section{SECONDARY FORESTS GROWING ON ABANDONED AGRICULTURAL FIELD IN THE PACIFIC REGION OF NICARAGUA. PROCEDURE FOR RESTORATION OF DEGRADED FOREST ECOSYSTEMS}

\section{Castro-Marín Guillermo, Gonzalez-Rivas Benigno}

Facultad de Recursos Naturales y del Ambiente, Universidad Nacional Agraria, Apartado postal 453, Managua, Nicaragua. E-mail: Guillermo.Castro@una.edu.ni, Benigno.Gonzalez@una.edu.ni

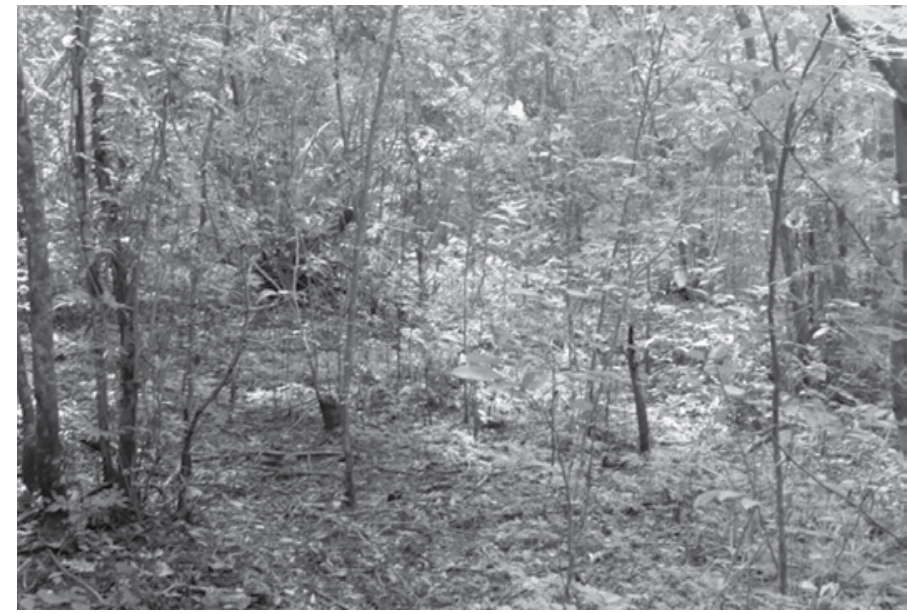

\section{RESUMEN}

En el presente estudio se determinó la composición de especie, diversidad y estructura de la población arbórea de tres bosques secundarios con edades de 4,9 y 14 años, derivados de tierras agrícolas abandonadas en la comarca La Chipopa-Rio Medina, Nandaime. Todos los individuos de igual altura, o con alturas superiores a $0.30 \mathrm{~m}$ de altura fueron identificados y medidos en 70 parcelas de $100 \mathrm{~m} 2$ en cada uno de los bosques bajo estudio. Un total de 12, 29 y 22 familias and subfamilias representadas por 17, 48 and 44 especies fueron registradas en los bosques de 4, 9 and 14 años, respectivamente. A través de la cronosecuencia de los bosques secundarios se registró un cambio en las especies dominantes, Lonchocarpus acuminatus registro el índice de mayor importancia en la estado sucesional de 4 años, Myrospermun frutescens y Guazuma ulmifolia tuvieron el más alto índice de valor de importancia en el estado de 9 años and Caesaeria corymbosa y Muntingia calabura, en el bosque de 14 años. La densidad y el área basal incrementaron al aumentar la edad de los bosques secundarios de 4 a 14 años. La diversidad de especie fue mayor en el bosque de 9 años seguido de 14 y 4-años. Se concluye que la recuperación de la vegetación arbórea sobre campos agrícolas abandonados

\section{ABSTRACT}

This study determined the species composition, diversity and population structure of three secondary forests recovered on abandoned agricultural fields after 4,9 and 14 years agricultural land in the community La Chipopa-Rio Medina, Nandaime. All stems $\geq 0.30 \mathrm{~m}$ height were identified and measured in 70 plots of $100 \mathrm{~m} 2$ in each of the secondary forests under study. A total of 12, 29 and 22 families and subfamilies represented by 17,48 and 44 species were recorded in the three secondary forests of 4, 9 and 14 years, respectively. There was a change in dominant species across of the different ages of the forest. Lonchocarpus acuminatus had the highest important value index in the forest of 4-year, Myrospermun frutescens and Guazuma ulmifolia had the highest importance value index in the 9 years forest, and Caesaeria corymbosa Muntingia calabura in the forest of 14 years. The density and basal area increased with increasing forest age from 4 to 14 years. The diversity of species was greater in forest of 9 years followed by 14 and 4 years. We conclude that the recovery of woody vegetation on abandoned agricultural fields is taking place satisfactory and is a suitable procedure for forest restoration, however, protection and conservation measures must be implemented to avoid natural or anthropogenic 
está marchando satisfactoriamente y es un procedimiento adecuado para la restauración forestal, sin embargo, medidas de protección y conservación deben de ser implementadas a fin de evitar perturbaciones naturales o antropogénicas que reviertan el proceso de sucesión. Se recomienda implementar medidas silviculturales como plantaciones de enriquecimiento que aceleren el proceso de recuperación y restauración de las áreas bajo estudio.

Palabras clave: diversidad, bosque seco, sucesión secundaria. disturbance that could reverse the process of succession. It is recommended to implement silvicultural measures such as enrichment planting to accelerate the recovery process and restoration of the areas under study.

Key words: forest diversity, dry forest, secondary succession
$\mathrm{E}$ 1 bosque tropical seco en uno de los ecosistemas forestales más amenazados y degradados en Centroamérica (Janzen, 2002). Históricamente este tipo de bosque han sido convertidos a otro tipo de uso de la tierra, como campos agrícolas, aéreas de pastizales, entre otras (Janzen, 1988; Brown y Lugo, 1990; Gillespie, 1999). Según Sabogal y Valerio (1998) el área de bosque que cubre actualmente toda la costa del pacifico de Mesoamérica representa menos del $2 \%$ de su área original, se estima que el área de bosque tropical seco es 3.4 millones de hectáreas (Janzen, 1988; Sabogal \& Valerio, 1998; Gillespie, 1999). Los remanentes de bosque tropical seco no son primarios, si no, un mosaico de formaciones forestales secundaria. (Kalacska, et al, 2004). En las últimas décadas muchas áreas boscosas que fueron convertidas a tierras agropecuarias, han sido abandonadas debido a cambios en intereses económicos, procesos de industrialización y urbanización (Thomlinson et al, 1996). Evidencias indican que áreas agrícolas abandonadas han sido recolonizadas rápidamente por comunidades vegetales a través de sucesiones secundarias, y el tiempo de colonización dependerá de intensidad de uso a que fue sometido antes de ser abandonada (Guariguata y Ostertag, 2001). Los bosques secos secundarios en los trópicos son una fuente importante de madera, productos forestales no maderable (Finegan, 1992; Chazdon y Coe, 1999) y proveen de servicios ambientales como protección de suelos, agua y fijación de carbono (Silver, et al., 2000). Asimismo, son importantes para la restauración de ecosistemas forestales y refugio de la biodiversidad en aéreas boscosas fragmentadas ( Lugo, 1992; Lamb, et al., 1997).

El presente estudio examina la dinámica de la colonización de la vegetación arbórea en terrenos que fueron sometidos a prácticas agrícolas y luego abandonado, el entendimiento de la dinámica de la sucesión secundaria, nos permite diseñar estrategias exitosas de restauración en áreas con vocación forestal que están actualmente degradadas (Peterson y Haines, 2000; Aide et al., 2000). No obstante, la mayoría de estudios en el neotrópico fueron realizados en el bosque húmedo tropical (Brown \& Lugo, 1990; Finegan, 1996;
Guariguata et al., 1997; Guariguata \& Ostergad, 2001; Peñas-Claros, 2003) y es muy limitada la información acerca del bosque seco.

El objetivo general de esta investigación fue examinar recuperación de la vegetación arbórea en bosques secundarios desarrollados en campos agrícolas abandonados en una cronosecuencia de estados sucesionales de 4, 9 y 14 años . Los objetivos específicos fueron describir la composición y diversidad florística de los diferentes bosques secundarios y determinar su estructura horizontal. Los resultados del presente estudio preverán información básica para el futuro manejo y restauración de bosques secundarios en el país.

\section{MATERIALES Y MÉTODOS}

Área de Estudio. El estudio se llevo a cabo en tres bosques secos secundarios en tres diferentes estados sucesionales localizados en la Comunidad "La ChipopaRio Medina" (11 ${ }^{\circ} 42^{\prime} 30^{\prime \prime} \mathrm{N}$ and $\left.86^{\circ} 05^{\prime} 30^{\prime \prime} \mathrm{W}\right)$, la cual está ubicada a 7 kilómetros de la ciudad de Nandaime, departamento de Granada (Figura 1). La vegetación en el área es clasificada como un bosque deciduo. La altitud varía entre 92 y 167 m.s.n.m. La media anual de presicipitación y temperatura es de $1444 \mathrm{~mm}$ and $27^{\circ} \mathrm{C}$, respectivamente. Los suelos predominantes en el área son vertisoles y alfisoles, originados de material volcánico y rocas básicas del periodo terciario (Rodríguez, et al., 2003).

En la década de los años 60, todos los bosques existentes en el área fueron cortados y las tierras fue utilizada para la producción de granos básicos (maíz, frijol y arroz) y pastizales para ganado. Recientemente, las áreas destinadas a agricultura y ganadería han sido abandonadas y paulatinamente han sido colonizadas por vegetación. Para la presente investigación se seleccionaron tres áreas abandonadas de 4, 9 y 14 años y con superficies de 1,3.7 and 4 hectáreas respectivamente con la asistencia de informante claves (Mario Soza y Carlos Soza, Nandarola; comunicación personal)

Métodos. La recolección de los datos se realizó a través de un inventario sistemático en cada uno de los bosques secundarios estudiados (4, 9 y 14 años). En cada bosque 
se establecieron 70 parcelas de $100 \mathrm{~m}^{2}(10 \mathrm{~m} \times 10 \mathrm{~m})$ siguiendo un diseño sistemático. En cada parcela de $10 \mathrm{~m} \times 10 \mathrm{~m}$ fueron establecidas aleatoriamente una subparcela de $5 \mathrm{~m}$ x $5 \mathrm{~m}$ y en una de la esquinas de esta, aleatoriamente se estableció una subparcela de $2 \mathrm{~m}$ x 2m (Sáenz y Finegan, 2000; Louman, et at., 2002). En las parcelas de $10 \mathrm{~m} \times 10 \mathrm{~m}$ fueron registrados toda la vegetación arbóreas $\geq 10 \mathrm{~cm}$ de diámetro normal ; en las parcelas de $5 \mathrm{~m} \times 5 \mathrm{~m}$ se midieron todos los individuos arbóreos entre $1.5 \mathrm{~m}$ altura a $9.9 \mathrm{~cm}$ de diámetro normal y en las parcelas de $2 \mathrm{~m} \times 2 \mathrm{~m}$ se registraron toda la vegetación arbórea entre $0.3 \mathrm{~m}$ a $1.5 \mathrm{~m}$ de altura . Los individuos fueron clasificados en diferentes clases de tamaño de vegetación, brinzales (individuos entre 0.3-1.5 $\mathrm{m}$ altura), latizales bajos (individuos entre $1.5 \mathrm{~m}$ altura a $4.9 \mathrm{~cm}$ de diámetro normal), latizal alto (individuos con $5 \mathrm{~cm}$ altura $\mathrm{y}<10 \mathrm{~cm}$ diámetro normal), fustales (individuos de $10 \mathrm{~cm}$ to $20 \mathrm{~cm}$ de diámetro normal and arboles remanentes (individuos $>20 \mathrm{~cm}$ de diámetro normal) (Sáenz and Finegan, 2000).

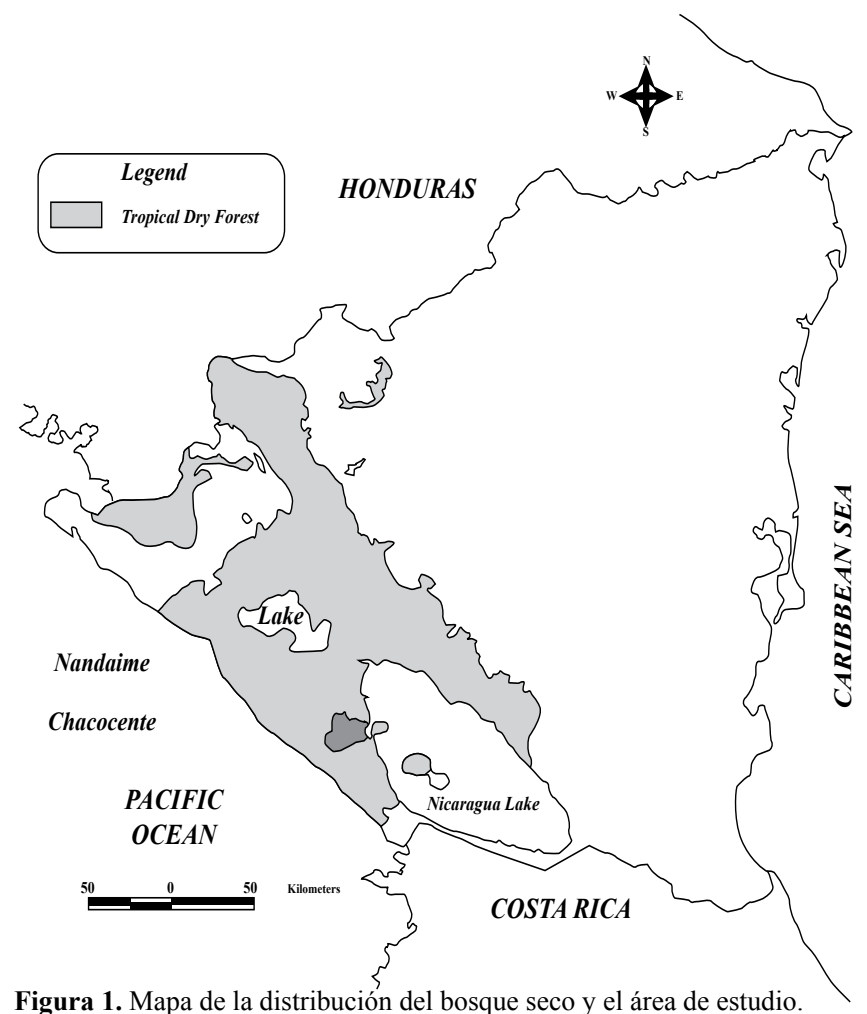

Análisis de los datos. Los siguientes índices y parámetros fueron utilizados para cada uno de los diferentes bosques secundarios:

1. Índice de diversidad de Shannon-Wiener (H') y su respectiva medición de igualdad (J') (Magurran, 1988).

$$
\begin{aligned}
& \mathbf{H}^{\prime}=-\sum_{(\mathbf{P i} \ln \mathbf{P i})} \\
& \mathbf{J}^{\prime}=\mathbf{H}^{\prime} / \ln \mathrm{S}
\end{aligned}
$$

Dónde:

H': Índice de diversidad de Shannon-Wiener.

Pi: La abundancia de la i-esima especie expresada como la proporción del total de individuos.

J': Índice de igualdad de Shannon-Wiener.

S: Es el número total de especies.

2. Estructura horizontal en términos de: (a) número de individuos por hectárea y su distribución en categorías diamétricas (para todos los individuos $\geq 1 \mathrm{~cm}$ diámetro normal) y (b) área basal (m2 ha-1) y su distribución por categoría diamétricas (individuos $\geq 1 \mathrm{~cm}$ diámetro normal)

3. El Índice de Valor de Importancia (IVI) fue empleado para describir la composición de especies en los diferentes bosques secundarios bajo estudio. El IVI de cada especie fue definido mediante la sumatoria de su dominancia relativa (DR), su abundancia relativa (AR) y su frecuencia relativa (FR), (Curtis and McIntosh, 1950) los cuales fueron calculados de la siguiente forma:

$\mathbf{D R}=$ Área basal total de una especie / el área basal total de todas las especies $\mathrm{x} 100$

$\mathbf{A R}=$ Número de individuos de una especie / el número total de individuos de totas las especies x 100

$\mathbf{F R}=$ frecuencia de una especie / suma de las frecuencias de totas las especies.

La frecuencia de una especie es definida como el número de parcelas en las cuales la especie está presente.

El rango teórico para dominancia, abundancia y frecuencia relativa es $0-100 \%$, por lo tanto, el IVI oscila entre 0 y $100 \%$.

El Índice de similaridad Jaccard de fue utilizado con el objetivo de examinar la similaridad florística entre los diferentes bosques secundarios utilizando la siguiente ecuación (Magurran, 1988):

$$
\mathbf{C} \mathbf{j}=\mathbf{j} /(\mathbf{a}+\mathbf{b}-\mathbf{j})
$$

Donde:

Cj: Indice de Similaridad de Jaccard

j: Es el número de especies encontradas en ambos sitios.

a: Es el número de especies encontradas en sitio A.

b: Es el número de especies encontradas en sitio B.

Debido a que muchas parcelas no se registraron individuos $\mathrm{y}$ esto hace difícil aplicar pruebas paramétricas, se ejecutaron pruebas de Kruskall-Wallis Test and Mann-Whitney U para comparar diversidad de especies, abundancia y area basal por clases de vegetación en los diferentes bosques secundarios estudiados. 


\section{RESULTADOS}

Composición florística. Un resumen de la composición de especies para los diferentes bosques secundarios es mostrado en la Tabla 1. Se registraron un total de 13, 29 y 22 familias en los bosques secundarios de 4, 9 y 14 años de edad, respectivamente. El número total de especies fue el doble en los bosques secundario de $9 \mathrm{y}$ 14 años comparados con el bosque de 4 años. El número de familia y especies se incrementa cuando el tamaño de la vegetación aumenta a través de la cronosecuencia (Tabla 2).
Cuatro familias y subfamilias en los bosques de 4 y 14 años y seis familias y subfamilias en el bosque de 9 años individualmente representaron más de $5 \%$ del total de los individuos (Figura 2). Entre esas familias, Flacourtiaceae y Papilionoideae fueron registradas en todos los bosques secundarios. Familias únicas para cada sitio están Theophrasteaceae en el bosque de 4 años, Boraginaceae and Sterculiaceae en el estado sucesional de 9 años y Apocynaceae en el de 14 años.

Tabla 1. Resumen de la composición florística y estructural de los bosques secundarios de 4, 9 y 14 años en Nandaime, Nicaragua

\begin{tabular}{|c|c|c|c|}
\hline \multirow[b]{2}{*}{ Características de la vegetación } & \multicolumn{3}{|c|}{ Edad del estado sucesional } \\
\hline & 4 años & 9 años & 14 años \\
\hline Familias & 13 & 29 & 22 \\
\hline Genero & 17 & 44 & 39 \\
\hline Epecies & 17 & 48 & 44 \\
\hline Densidad indiv- $\mathrm{ha}^{-1}$ & 5011 & 6914 & 1067 \\
\hline 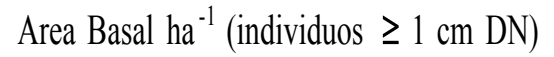 & 1.49 & 9.17 & 10.67 \\
\hline
\end{tabular}

Tabla 2. Características florística y estructural de los bosques secundarios desarrollados en campos agrícolas abandonados por clase de tamaño de la vegetación, Nandaime

\begin{tabular}{lcccccc}
\hline Clase & $\begin{array}{c}\text { Edad del } \\
\text { estado } \\
\text { sucesional }\end{array}$ & $\begin{array}{c}\mathrm{N}^{\circ} \\
\text { Familias }\end{array}$ & $\begin{array}{c}\mathrm{N}^{\circ} \\
\text { Especies }\end{array}$ & Densidad & $\begin{array}{c}\text { Abundancia } \\
\text { Relativa }\end{array}$ & $\begin{array}{c}\text { Área } \\
\text { basal }\end{array}$ \\
\hline Brinzal & 4 & 8 & 10 & 2000 & 39.9 & $*$ \\
& 9 & 14 & 20 & 3000 & 43.4 & $*$ \\
\hline Latizal & 14 & 14 & 22 & 5786 & 60.0 & $*$ \\
Bajo & 4 & 10 & 13 & 2914 & 58.2 & 1.49 \\
& 9 & 19 & 30 & 3011 & 43.5 & 2.89 \\
Latizal & 14 & 18 & 28 & 2011 & 20.9 & 2.09 \\
Alto & 4 & 6 & 6 & 97 & 1.9 & 0.82 \\
& 9 & 21 & 29 & 771 & 11.2 & 2.77 \\
Fustal & 14 & 19 & 28 & 1640 & 17.0 & 5.66 \\
& 9 & 0 & 0 & 0 & 0.0 & 0.00 \\
& 14 & 13 & 16 & 103 & 1.5 & 1.51 \\
Arboles & 4 & 0 & 0 & 187 & 1.9 & 2.40 \\
Remanentes & 9 & 7 & 8 & 0 & 0.0 & 0.00 \\
& 14 & 5 & 5 & 7 & 0.4 & 2.00 \\
\hline
\end{tabular}

Área basal para brinzales no fue calculado. 
En la categoría de vegetación de brinzalez, cinco especies en el estado sucesional de 4 y 14 años, y seis especies en el de 9 años, individualmente registraron más del $5 \%$ del total de todos los individuos Dentro de esas especies, Caesaeria corymbosa Kunth. y Lonchocarpus acuminatus (Schltdl.) M. Souza fueron encontradas en todos los bosques secundarios. Mientas que Jacquinia aurantiaca Aiton., Diospyros nicaraguensis Standl. y Myrospermum frutescens Jacq. fueron registradas exclusivamente en el estado sucesional de 4-años, Guazuma ulmifolia Lam. y Cordia bicolor A.DC. estuvieron presentes en el de 9 años, y Lonchocarpus minimiflorus Donn. Sm. fue encontrado exclusivamente en el estado sucesional de 14años.

En la clase de vegetación de latizales bajos y altos, L. acuminatus fue la especies más importante en latizales bajos y Hippocratea rosea $L$ en latizal alto en el estado sucesional de 4 años, (Tabla 3). En el estado sucesional de 9 años de abandono, M. frutescens and A. costaricensis fueron de mas importante especies de latizales bajos, G. ulmifolia y L. acuminatus en latizales altos, G. ulmifolia y Caesalpinia exostemma DC en la categoría de vegetación de latizales y Cordia alliodora (Ruiz \& Pav.) Oken e Inga spuria (Willd.) J. en la clase de vegetación de árboles remanentes (Tabla 4). En el estado sucesional de 14 años de abandono, las especies más importante fueron $C$. corymbosa y $S$. obovata en latizales bajos, Muntingia calabura L. y L. acuminatus en latizal alto, Gliricidia sepium Kunth. ex Steud. Y L. acuminatus en fustales, y Tabebuia rosea (Bertol.) A. DC. Y Cassia grandis L. f. en la clase de vegetación de árboles remanente (Tabla 5). Tres especies, $M$. frutescens y C. corymbosa (latizal bajo) and L. acuminatus (latizal alto), fueron registradas en todos los tres estados sucesionales de los bosques secundarios. Entre la cinco especies más importantes en la clase de vegetación de árboles remanentes solamente G. ulmifolia, fue registrada en todas la categorías de vegetación (brinzal, fustales y latizales) en el bosque secundario de 9 años.
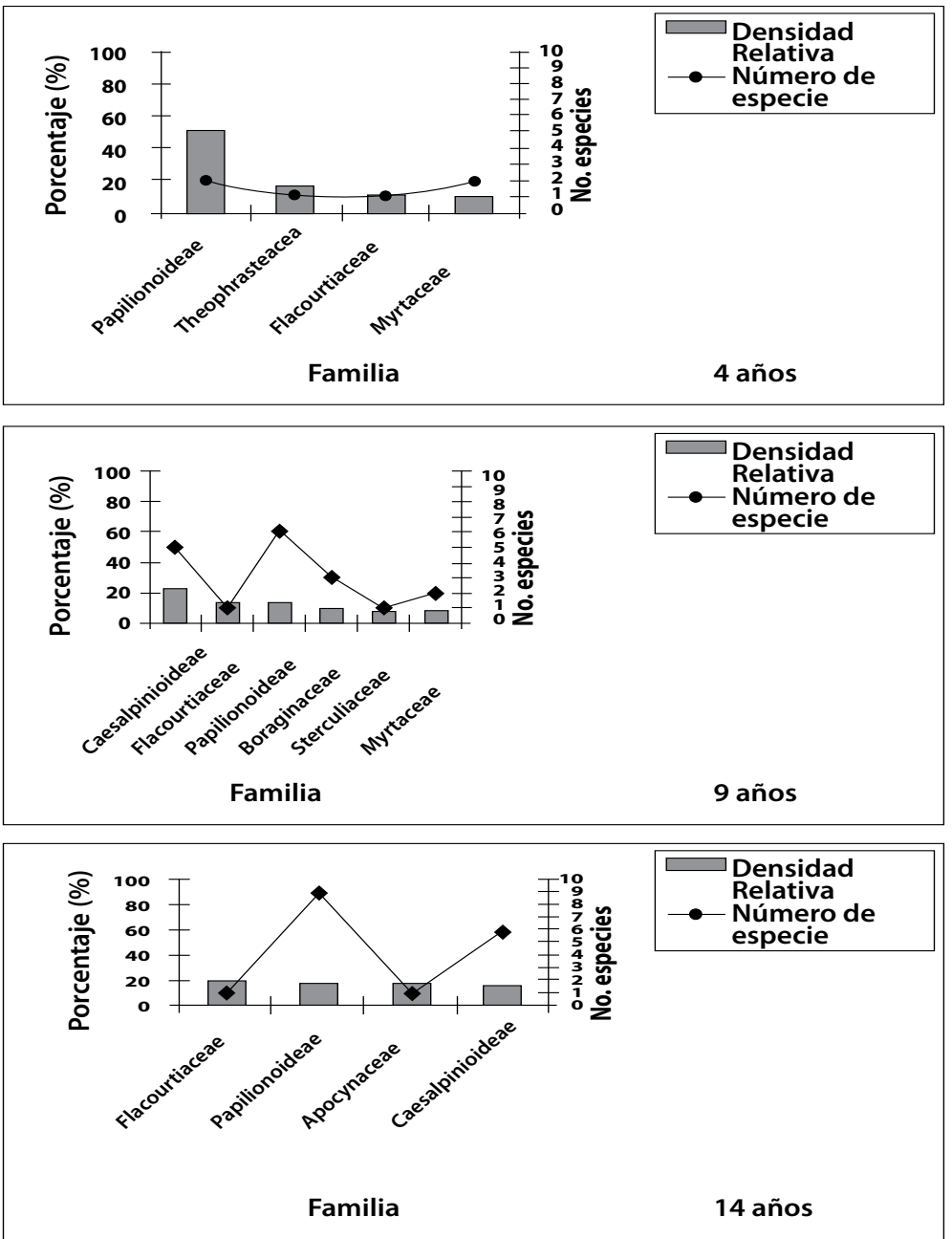

Figura 2. Familias y subfamilias con densidades superiores al 5\% del total del número de individuos en los bosques secundarios desarrollados en campos agrícolas abandonados en el Pacifico de Nicaragua. 
Tabla 3. Índice de Valor de Importancia (IVI) de la cinco especies más abundantes en el bosque secundario de 4 años de edad

\begin{tabular}{llcrrr}
\hline $\begin{array}{l}\text { Clase de } \\
\text { tamaño }\end{array}$ & Species & $\begin{array}{c}\text { Abundancia } \\
\text { relativa }\end{array}$ & $\begin{array}{c}\text { Dominancia } \\
\text { relativa }\end{array}$ & $\begin{array}{c}\text { Frecuencia } \\
\text { relativa }\end{array}$ & IVI \\
\hline \multirow{2}{*}{ Brinzal } & Lonchocarpus acuminatus & 63.1 & 67.8 & 36.9 & 167.8 \\
& Myrospermun frutescens & 17.1 & 12.8 & 26.4 & 56.2 \\
& Caesaeria corymbosa & 6.9 & 7.9 & 13.2 & 28.0 \\
& Hippocratea rosea & 2.9 & 4.4 & 5.3 & 12.6 \\
& Jacquinia aurantiaca & 5.5 & 4.2 & 1.3 & 11.0 \\
\hline \multirow{2}{*}{ Latizal } & Lonchocarpus acuminatus & 58.8 & 83.6 & 37.0 & 179.4 \\
& Hippocratea rosea & 11.8 & 4.6 & 18.8 & 35.2 \\
& Acacia costaricensis & 11.8 & 2.7 & 18.8 & 33.3 \\
& Myrospermum frutescens & 5.9 & 4.0 & 8.4 & 18.3 \\
& Dalbergia retusa & 5.9 & 3.7 & 8.4 & 18.1 \\
\hline
\end{tabular}

Tabla 4. Índice de Valor de Importancia (IVI) de la cinco especies más abundantes en el bosque secundario de 9 años de edad

\begin{tabular}{|c|c|c|c|c|c|}
\hline $\begin{array}{c}\text { Clase de } \\
\text { tamaño }\end{array}$ & Species & $\begin{array}{c}\text { Abundancia } \\
\text { relativa }\end{array}$ & $\begin{array}{l}\text { Dominancia } \\
\text { relativa }\end{array}$ & $\begin{array}{c}\text { Frecuencia } \\
\text { relativa }\end{array}$ & IVI \\
\hline \multirow{5}{*}{$\begin{array}{l}\text { Latizal } \\
\text { Bajo }\end{array}$} & Myrospermum frutescens & 10.2 & 32.2 & 8.6 & 51.0 \\
\hline & Acacia costaricensis & 15.7 & 12.2 & 9.6 & 37.5 \\
\hline & Caesaeria corymbosa & 10.8 & 5.9 & 10.2 & 27.0 \\
\hline & Diospyros nicaraguensis & 9.9 & 7.8 & 8.6 & 26.2 \\
\hline & Lonchocarpus acuminatus & 7.6 & 6.4 & 8.6 & 22.6 \\
\hline \multirow{5}{*}{$\begin{array}{l}\text { Latizal } \\
\text { alto }\end{array}$} & Guazuma ulmifolia & 18.5 & 22.1 & 13.5 & 54.2 \\
\hline & Lonchocarpus acuminatus & 16.3 & 13.2 & 9.5 & 39.0 \\
\hline & Karwinskia calderonii & 5.9 & 7.1 & 2.7 & 15.8 \\
\hline & Eugenia salamensis & 5.9 & 4.4 & 5.4 & 15.7 \\
\hline & Pissonia macranthocarpa & 4.4 & 5.5 & 5.4 & 15.3 \\
\hline \multirow[t]{5}{*}{ Fustal } & Guazuma ulmifolia & 31.9 & 30.2 & 31.9 & 94.0 \\
\hline & Caesalpinia exostemma & 23.6 & 23.6 & 13.7 & 60.9 \\
\hline & Cordia bicolor & 9.7 & 8.5 & 11.3 & 29.5 \\
\hline & Cochlospermun vitifolium & 8.3 & 9.4 & 9.1 & 26.9 \\
\hline & Myrospermum frutescens & 4.2 & 6.6 & 4.6 & 15.4 \\
\hline \multirow{5}{*}{$\begin{array}{l}\text { Árboles } \\
\text { remanentes }\end{array}$} & Cordia alliodora & 25 & 34.3 & 36.8 & 96.1 \\
\hline & Inga spuria & 20 & 18.6 & 9.0 & 47.6 \\
\hline & Guazuma ulmifolia & 20 & 12.9 & 9.0 & 41.9 \\
\hline & Cassia grandis & 15 & 12.1 & 9.0 & 36.2 \\
\hline & Swietenia humilis & 5 & 7.9 & 9.0 & 21.9 \\
\hline
\end{tabular}

Diversidad y similaridad. El patrón de distribución especie-abundancia del bosque secundario originado de campos agrícolas abandonados muestran una distribución de J invertida o distribución de series log. La mayoría de las especies poseen muy pocos individuos, mientras que pocas especies están representados por muchos individuos en todos los bosques secundarios bajo estudio.

El índice de diversidad de Shannon-Wiener, es presentado en la Tabla 6 para cada bosque secundario y por clase de tamaño de vegetación. La diversidad de especies vario significativamente entre los diferentes bosques secundarios, para brinzales (Kruskall-Wallis Test $x 2=13.201 ; \mathrm{p}=0.001$ ), latizal bajo (Kruskall-Wallis Test $x 2=34.291 ; \mathrm{p}<0.0001)$ y latizal alto (KruskallWallis Test $\left.x_{2}=55.224 ; \mathrm{p}<0.0001\right)$. La diversidad de especie de las categorías de tamaño de la vegetación de fustales y arboles remanentes vario significativamente entre los bosques secundarios de 9 y 14 años (MannWhitney U test; $\mathrm{p}=0.046$ )

La similaridad en composición de especies entre los diferentes bosques secundarios es mostrada en la Tabla 7. Agrupando todas las especies de las diferentes clases de vegetación, la similaridad en composición de especies fue más alta entre los bosques secundarios de 9 y 14 años de abandono. Al comparar la similaridad en la composición de especies entre las diferentes clases de tamaño de la vegetación, encontramos que los latizales bajo y alto de los bosques secundarios de 9 y 14 años poseen una considerable similaridad de especies. Sin embargo, los latizales bajos de 4 y 14 años tienen una alta similaridad de especies en comparación el bosque secundario de 9 años. 
Tabla 5. Índice de Valor de Importancia (IVI) de la cinco especies más abundantes en el bosque secundario de 14 años de edad

\begin{tabular}{|c|c|c|c|c|c|}
\hline $\begin{array}{c}\text { Clase de } \\
\text { tamaño }\end{array}$ & Species & $\begin{array}{l}\text { Abundancia } \\
\text { relativa }\end{array}$ & $\begin{array}{l}\text { Dominancia } \\
\text { relativa }\end{array}$ & $\begin{array}{l}\text { Frecuencia } \\
\text { relativa }\end{array}$ & IVI \\
\hline \multicolumn{6}{|l|}{ Latizal } \\
\hline \multirow[t]{5}{*}{ bajo } & Caesaeria corymbosa & 30.1 & 31.6 & 2.6 & 64.4 \\
\hline & Stemmadenia abovata & 21.0 & 21.7 & 5.8 & 48.5 \\
\hline & Acacia costaricensis & 7.1 & 4.4 & 10.0 & 21.6 \\
\hline & Myrospermum frutescens & 4.5 & 5.2 & 3.2 & 13.0 \\
\hline & Tabebuia rosea & 0.9 & 0.8 & 10.7 & 12.3 \\
\hline \multicolumn{6}{|l|}{ Latizal } \\
\hline \multirow[t]{5}{*}{ alto } & Muntingia calabura & 24.7 & 20.1 & 19.2 & 64.1 \\
\hline & Lonchocarpus acuminatus & 13.2 & 16.9 & 11.3 & 41.4 \\
\hline & Stemmadenia abovata & 12.9 & 11.8 & 11.9 & 36.6 \\
\hline & Myrospermum .frutescens & 10.8 & 12.0 & 11.3 & 34.1 \\
\hline & Acacia costaricensis & 6.6 & 6.7 & 8.6 & 21.9 \\
\hline \multicolumn{6}{|l|}{ Fustal } \\
\hline & Gliricidia sepium & 31.3 & 34.9 & 23.8 & 90.0 \\
\hline & Lonchocarpus acuminatus & 15.3 & 12.6 & 17.6 & 45.5 \\
\hline & Guazuma ulmifolia & 11.5 & 12.0 & 12.6 & 36.0 \\
\hline & Myrospermum .frutescens & 9.2 & 6.6 & 7.5 & 23.2 \\
\hline & Diphysa robinioides & 6.9 & 6.1 & 5.0 & 18.0 \\
\hline \multicolumn{6}{|l|}{ Árboles } \\
\hline \multirow[t]{5}{*}{ remanentes } & Tabebuia rosea & 20 & 37.9 & 20 & 77.9 \\
\hline & Casia grandis & 20 & 25.6 & 20 & 65.6 \\
\hline & Gliricidia sepium & 20 & 16.8 & 20 & 56.8 \\
\hline & Chomelia spinosa & 20 & 10.9 & 20 & 50.9 \\
\hline & Cordia alliodora & 20 & 8.8 & 20 & 48.8 \\
\hline
\end{tabular}

Tabla 6. Índice de Diversidad de Shannon-Wiener para los diferentes bosques secundarios desarrollados en campos agrícolas abandonados por clase de tamaño de vegetación

\begin{tabular}{lccc}
\hline & \multicolumn{3}{c}{ Edad del estado sucesional } \\
Clase de tamaño & 4 años & 9 años & 14 años \\
\hline Brinzal & 1.87 & 2.41 & 2.40 \\
Latizal bajo & 1.26 & 2.85 & 2.43 \\
Latizal alto & 1.32 & 2.87 & 2.53 \\
Fustal & 0.00 & 2.19 & 2.30 \\
Árboles remanentes & 0.00 & 1.87 & 1.61 \\
\hline
\end{tabular}

Tabla 7. Índice de similaridad de Jaccard por clase de tamaño de vegetación en los diferentes bosques secundarios desarrollados en campos agrícolas abandonados

\begin{tabular}{lcc}
\hline Clase de tamaño & Edad comparada & Índice de Jaccard's (\%) \\
\hline \multirow{2}{*}{ Brinzal } & 4 vrs 9 & 20.0 \\
& 9 vrs 14 & 23.5 \\
& 4 vrs 14 & 33.3 \\
\hline \multirow{2}{*}{ Latizal bajo } & 4 vrs 9 & 26.5 \\
& 9 vrs 14 & 44.0 \\
& 4 vrs 14 & 24.2 \\
\hline Latizal alto & 4 vrs 9 & 16.7 \\
& 9 vrs 14 & 39.0 \\
& 4 vrs 14 & 13.3 \\
\hline Fustal & 9 vrs 14 & 27.6 \\
\hline Árboles remanentes & 9 vrs 14 & 18.2 \\
\hline
\end{tabular}


Estructura. La densidad de total incremento de 5011 a 9629 individuos por ha a cuando la edad del abandono aumentada de 4 a 14 años (Tabla 1). Una tendencia similar en densidad se observo las clases de vegetación, brinzal, latizale altos y fustales a través de los diferentes bosques secundarios. Sin embargo, la densidad de latizales bajo fue mayor en los bosques secundarios de 4 y 9 años comparado con el de 14 años (Tabla 2).

La prueba de Kruskal-Wallis revelo diferencias significativas en densidad entre los diferentes estados sucesional para brinzal $(x 2=1.00 ; \mathrm{P}=0.001)$, latizal bajo $(x 2=7.83 ; \mathrm{p}=0.02)$ and latizal alto $(x 2=101.50, \mathrm{p}$ $<0.0001)$. La densidad de la clase de vegetación fustal y árboles remanentes también fue significativamente diferente entre los bosques secundarios de 9 y 14 años (Mann-Whitney U test; $p=0.046$ para ambas clases)

El área basal total de los individuos $\geq 1 \mathrm{~cm}$ de diámetro normal, también se incrementó con la edad de los diferentes bosques secundarios (Tabla 1). Se encontró diferencias significativas en área basal de latizales bajos $(x 2=19.124 ; \mathrm{p}<0.0001)$, latizales altos $\left(x_{2}=97.059 ; \mathrm{p}<0.0001\right)$, fustales $\mathrm{y}$ árboles remanentes (Mann-Whitney U test; $\mathrm{p}=0.046$ )

La distribución de los individuos y el área basal en relación a las clases diamétricas son mostradas en la figura 3. Para ambos bosques secundarios de 9 y 14 años la distribución de los individuos a través de las categorías diamétricas fue de una $\mathrm{J}$ invertida, sugiriendo que los individuos de más pequeño tamaño son los más abundantes La distribución del área basal en las categorías diamétricas, mostraron que en las dos primeras clases se concentran más del $60 \%$ del área basal total de los individuos $\geq 1 \mathrm{~cm}$ de diámetro normal en los bosques secundarios de 9 y 14 años. No se registraron individuos $\geq 10 \mathrm{~cm}$ en el bosque secundario de 4 años, pero los individuos con menores tamaños de diámetro fueron los más abundantes y en la categoría díamétrica de 5-9.9 cm de diámetro se concentró la mayor cantidad de área basal.
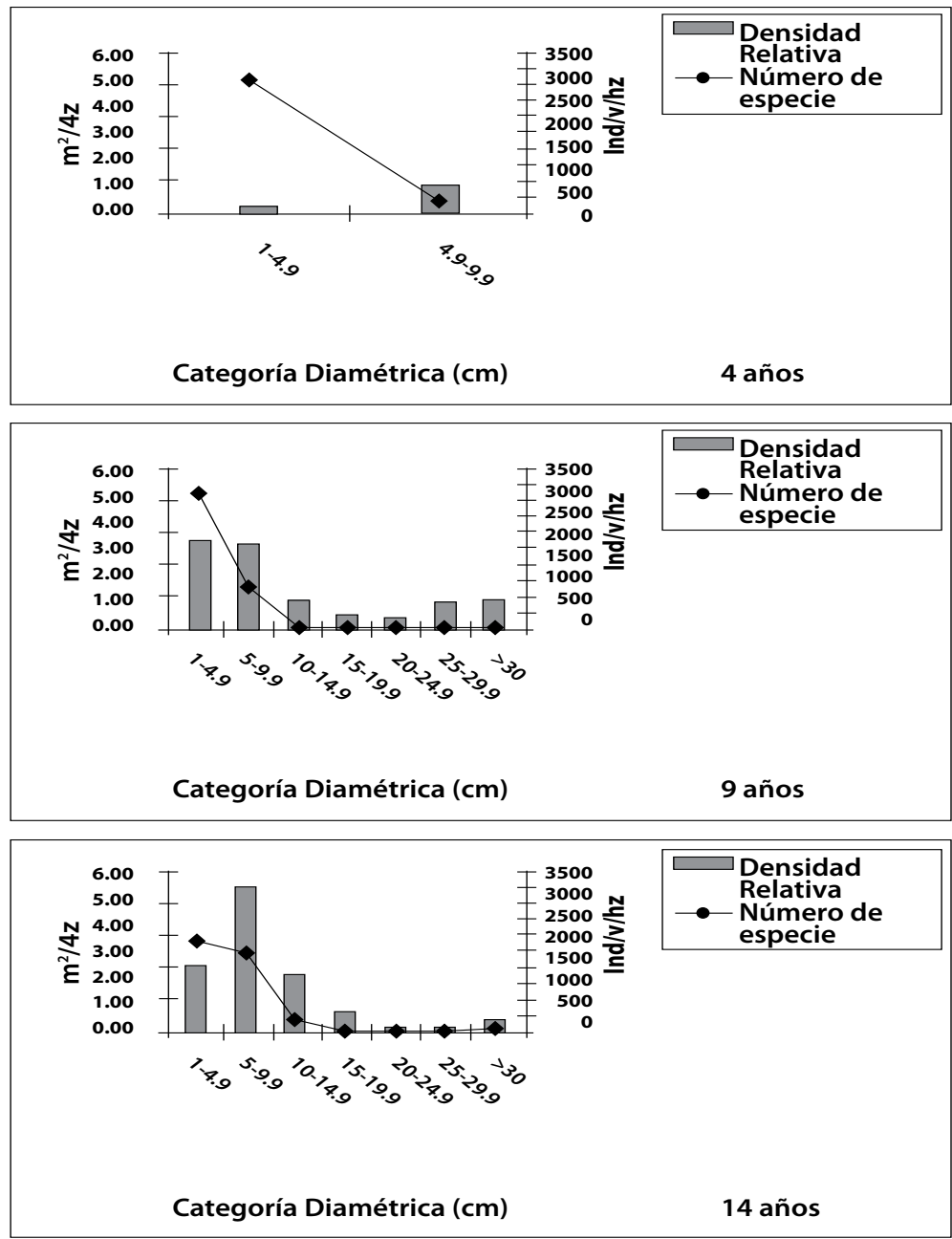

Figura 3. Distribución de la densidad y el área basal de los individuos $\geq 1 \mathrm{~cm}$ de diámetro normal en relación con las categorías diamétricas recuperada después de 4, 9 y 14 años de abandono. 


\section{DISCUSIÓN}

Los resultados del presente estudio indican que la recuperación de la composición florística avanza satisfactoriamente al aumentar la edad de los bosques secundarios. Cuando la edad de la sucesión avanza incrementa el número de familias y especie. Por ejemplo, en el bosque secundario de 4 años, 3 familias y subfamilias contienen el $80 \%$ de los individuos registrados. En los bosques secundarios de mayor edad 9 y 14 años, el $87 \%$ del total de los individuos están representados en 12 y 10 familias y subfamilias respectivamente. Es importante destacar que hubo un cambio en dominancia en las familias y subfamilias, a través de los diferentes bosques secundarios de los bosques secundarios. Fabaceae-papilionoideae es dominate en el bosque secundario de 4 años, mientras que Fabaceae-caesalpinioideae and Flacourtiaceae dominan en los bosques secundarios de 9 y 14 años, respectivamente. Las especies dominantes por clases de tamaño de vegetación también son diferentes a través de los diferentes bosques secundarios evaluados, indicando que hay algunas especies que dominan según la edad de la sucesión. Lo anterior concuerda con el patrón general de la recuperación florística y estructural que presentan las sucesiones en una cronosecuencia. Durante la primera etapa de la colonización, la vegetación es dominada por hierbas, arbustos, lianas, los cuales tienen una vida corta y especies arbóreas pioneras tales como: H. rosea y J. aurantiaca la cuales fueron registradas en nuestro estudio. Después de este periodo, el dosel es dominado por especies heliófilas durables (Diphysa robinioidies and Gliricidia sepium registradas en el estudio), y finalmente el dosel del bosque secundario es remplazado por especies esciofitas parciales y totales (Guariguata y Ostertag, 2001). La baja similaridad entre el bosques secundarios de 4 años con los de edad más avanzada, proveen evidencia acerca de los cambios en composición de especies a medida que la sucesión avanza en edad.

La recuperación de la riqueza de especie en una sucesión secundaria depende de una variedad de factores tales como: intensidad de uso de la tierra, disponibilidad de semillas, agentes dispersores, así, también como factores abióticos (luz, humedad, propiedades físicas y química de los suelos). Se considera que cuando el terreno abandonado fue sometido a un uso moderado y existen fuentes cercanas de semillas, la recuperación de la riqueza de especies arbóreas en una sucesión secundaria ocurre con mayor rapidez y en varias décadas alcanza valores en estructura y composición florística de un bosque maduro (Guariguata and Ostertag, 2001). La presencia de árboles remanentes en las áreas de estudio juegan un importante rol en la colonización de vegetación del los sitios, ya que sirven como hábitat y son fuentes de alimentación para los agentes dispersores de semillas (Guevara et al., 1986; da Silva et al., 1996;
Holl et al., 2000). En nuestro estudio registramos 29 árboles remanentes por hectárea en el bosque secundario de 9 años distribuidos entre 8 especies y 7 individuos de cinco especies en el bosque secundario de 14 años. Algunas especies de árboles remanentes, tal es el caso, de G. ulmifolia , se encuentra abundantemente en la clase de vegetación de latizal tanto bajo como alto. Aparentemente, la relativa mayor riqueza de especies en los bosques secundarios de 9 y 14 años podría ser atribuido a una mejor condición de dispersión de semillas. En términos generales, la variación en la composición de especies está relacionado a los cambios en la condiciones ambientales que ocurren a medida que avanza transcurre el tiempo de la sucesión y a las diferencias entre la especies con respecto a las condiciones de establecimiento que ellas requieren, y a la estrategia de vida durante su ciclo de vida.

El patrón de distribución especie -abundancia muestra que la mayoría de la especies en todos los bosques secundarios bajo estudio están representadas por muy pocos individuos, mientras que pocas especies están representadas por muchos individuos, una característica típica de bosques maduros en el trópico (Huang et al., 2003; Pitman et al., 1999). Muchos estudios han mostrado que la diversidad de especies aumenta con la edad de la sucesión (Perkulis et. al., 1997; Kennard, 2002; Peña-Claros, 2003; Kalacska, et al., 2004) al igual que en nuestro estudio, que los rangos de los valores de los índices de Shannon-Wiener oscilan entre 1.5 and 3.5 y raramente sobrepasan el valor de 5 (Magurran, 2004).

La estructura horizontal (densidad y área basal) y la diversidad de especie mostro aumentos significativos cuando la sucesión alcanza mayor edad. Asimismo, encontramos marcadas diferencias en la estructura y diversidad entre las diferentes clases de tamaño de la vegetación. La densidad de los brinzales incremento consistentemente al aumentar la edad del abandono. Esto indica que el avance de la sucesión crea condiciones favorables para el establecimiento y desarrollo de los individuos, lo cual se considera como una de la principales barrera para la recuperación de bosques secundarios en campo abandonados (Zimmerman et al., 2000; Wijdeven y Kuzee, 2000; Slocum, 2000)

La densidad y el área basal de latizales bajos declino cuando la edad del abandono incremento de 9 a 14 años. Esto podría estar relacionado con el cierre del dosel, lo cual limita el establecimiento y desarrollo de especies heliófilas efímeras debido a la sombra (Finegan, 1996; Whitmore, 1998). Lo anterior puede ser comprobado, ya que el número de latizales altos y fustales es mayor en el bosque secundario de 14 años en comparación con el de 9 años.

Es importante señalar que la ausencia de bosques maduros cerca de nuestra área de estudio fue un limitación para realizar comparaciones entre los bosques 
secundarios derivados de campos abandonados y bosque maduros. Sin embargo, en un intento por comparar el proceso de recuperación se tomo como referencia el bosque seco deciduo de Chacocente, localizado a 30 $\mathrm{Km}$ de nuestra área de estudio. Según González, et al. (2006) el bosque maduro del refugio de vida Silvestre de Chacocente está compuesto de 29 familias y 59 especies para árboles $\geq 10 \mathrm{~cm} \mathrm{dbh}$, categorías de tamaño de vegetación referida en nuestro estudio como latizales y árboles remanentes. Comparando con estos resultados, la composición florística del bosque secundario de 14 años se recupera rápidamente (15 familias and 21 especies). Castro et. al, (2005) en el mismo bosque seco de Chacocente, reporto 450 individuals/ha with a basal área of $15.62 \mathrm{~m} 2 / \mathrm{ha}$. Aparentemente la recuperación del estado sucesional de 14 años de edad es relativamente lenta ( densidad $=187$ ind. $/$ ha; basal area $=2.40 \mathrm{~m} 2 /$ ha). Se asume, que esta baja recuperación en estructura puede estar asociado con la disponibilidad de nutrientes. Tucker, et al.(1988)

En términos generales nuestros resultados concuerdan con estudios previos sobre bosque secos secundarios en Costa Rica (Kalacska et al., 2004), plantaciones de henequén abandonadas en Mexico (Perkulis et al., 1997), agricultura migratoria en Bolivia ((Kennard, 2002), sucesiones secundarias en la amazonia boliviana Amazon (Peña-Claros, 2003)

\section{CONCLUSIÓN}

El desarrollo de bosques secundarios sobre campos agrícolas abandonados es alentadora para la posibilidad de manejo restauración de los bosques secundarios tropicales. Actualmente, los objetivos de manejo de estos parches boscosos no han sido definidos, y no se han planificado ningún tipo de intervención silvicultural , ya sea para conservación o producción. Por lo tanto, un plan de manejo debe ser elaborado en consulta con los intereses de los dueños de la propiedad. Se recomiendan tomar medidas de protección de las áreas, ya que cualquier tipo de perturbación de tipo natural (fuego) o antropogenico (pastoreo, tala para leña u otra necesidad) puede revertir significativamente el proceso de colonización vegetal sobre las áreas de estudio. Asimismo, con el objetivo de acelerar el proceso de recuperación, plantaciones de enriquecimiento con especies heliófilas durables o esciofitas deben ser llevadas a cabo.

\section{REFERENCIAS BIBLIOGRÁFICAS}

Aide, TM; Zimmerman, JK, Pascarella, JB, Rivera, L, Marcano-Vega, H. 2000. Forest Regeneration in Chronosequence of Tropical Abandoned Pasture: Implications for Restoration Ecology. Restor. Ecol. 8, 328-338.

Brown, S; Lugo, AE. 1990. Tropical secondary forest. J. Trop. Ecol. 6, 1 - 32.

Castro, G; Nygård, R; Gonzales, B; Oden, PC. 2005. Stand dynamics and basal area change in a tropical dry forest reserve in Nicaragua. For. Ecol. Manage. 208, 63-75.

Chazdon, RL; Coe, FG. 1999. Ethnobotany of woody species in second growth, old-growth, and selectively logged forests of Northeastern Costa Rica. Conserv. Biol.13, 1312-1322.

Curtis, JT; McIntosh, RP. 1950. The interrelation of certain analytic and synthetic phytosociological characters. Ecology. 34, 741-757.

da Silva, JMC; Uhl, C, Murray, G. 1996. Plant succession, landscape management, and the ecology of frugivorous birds in abandoned amazonian pastures. Conserv. Biol. 10, 491-503.

Finegan, B. 1992. The management potential of neotropical secondary lowland rain forest. For. Ecol. Manage. 47, 295-321.

Finegan, B. 1996. Pattern and process in neotropical secondary rain forest: the first hundred years of succession. Trends. Ecol. Evol. 11, $119-124$.

Gillespie, TW. 1999. Life history characteristics and rarity of wood plants in tropical dry forest fragments of Central America. J.Trop. Ecol. 15, 637 - 649.

González, B; Tigabu, M; Gerhardt, K; Castro, G; Odén, PC. 2006. Species composition, diversity and local use of tropical dry deciduous and gallery forests in Nicaragua. Biodiversity and Conservation. 15: 15091527 
Guariguata, MR; Chazdon, RL; Denslow, JS; Dupuy, JM; Anderson, L. 1997. Structure and floristic of secondary and old-growth forest stands in lowland Costa Rica. Plant. Ecol. 132, 107-120.

Guariguata, MR; Ostertag, R. 2001. Neotropical secondary succesion: changes in structural and functional characteristics. For. Ecol. Manage. 148, 185-206.

Guevara, S; Purata, SE; Van der Maarel, E. 1986. The role of remnant forest tress in tropical secondary sucesión. Vegetatio. 66, 77-84.

Holl, KD; Loik, ME; Lin, EHV; Samuels, IA. 2000. Tropical montane forest restoration in Costa Rica: Overcoming barriers to dispersal and establishment. Restor. Ecol. 8, 339-349.

Huang, W; Pohjonen, V; Johansson, S; Nashanda, M; Katigula, MIL; Luukkanen, O. 2003. Species diversity, forest structure and species composition in Tanzanian tropical forests. For. Ecol. Manage. 173, 11-24.

Janzen, D. 1988. Management of habitat fragments in a tropical dry forest: growth. Ann. Mo. Bot. Gard. 75, 105-116

Janzen, D. 2002. Tropical dry forest: Area de Conservación Guanacaste, northwestern Costa Rica. In: Perrow M.R. and Davy A.J. (Eds.), Handbook of Ecological Restoration, volume 2 Restoration in Practice. Cambridge University Press, Cambridge, p. 559-583.

Kalacska, M; Sanchez-Azofeifa, GA; Calvo-Alvarado, JC; Quesada, M; Rivard, B; Janzen, DH. 2004. Species composition, similarity and diversity in three successional stages of a seasonally dry tropical forest. For. Ecol. Manage. 200, 227-247.

Kennard, DK. 2002. Secondary forest succession in a tropical dry forest: patterns of development across a 50-year chronosequence in lowland Bolivia. J. Trop. Ecol. 18, 53-66.

Lamb, D; Parrota, J; Keenan, R, Tucker, N. 1997. Rejoining habitat remnants: restoring degraded rainforest lands. In: Laurance, W.F., Bierregaard Jr., R.O. (eds), Tropical Forest Remnants. University of Chicago Press, Chicago, IL, pp. 366-385.

Louman, B; Mejía, A; Núñez, L. 2002. Inventario en bosques secundarios. In. Orozco, L. and Brumér, C. (eds) Inventario Forestales para Bosques Latifoliados en América Central, pp. 171 - 216. CATIE, Turrialba, Costa Rica.

Lugo, AE. 1992. Comparision of tropical tree plantation and with secondary forest of similar age. Ecological Monographs 62, 1-41

Magurran, AE. 1988. Ecological Diversity and its Measurements. University Press. Cambridge. London. p 179.

Magurran, AE. 2004. Measuring Biological Diversity, Blackwell Science, Malden, MA, USA 256 pp.

Peña-Claros, M. 2003. Changes in forest structure and species composition during secondary succession in the Bolivian Amazon. Biotropica. 35, 450-461.

Perkulis, AM; Ramos Prado, JM; Jiménez-Osornio, JJ. 1997. Composition, structure and management potencial of secondary dry tropical vegetation in two abandoned henequen plantations of Yucatan, Mexico. For. Ecol. Manage. 94, 79-88.

Pitman, NCA; Terbirgh, J; Silman, MR; Nuñez, VP. 1999. Tree species distribution in a upper Amazonian forest. Ecology 80, 2651-2661.

Peterson, CJ; Haines, BL. 2000. Early Successional Patterns and Potential Facilitation of Woody Plan Colonization by Rotting Logs in Premontane Costa Rican Pasture. Restor. Ecol. 8, 361-369.

Rodríguez, I; Aguirre, C; Mendoza B. 2003. Actualización del Estado del recurso suelo y capacidad de uso de la tierra de los municipios de Santa Teresa y Nandaime. Facultad de Recursos Naturales y del Ambiente, Universidad Nacional Agraria. Proyecto Sur-Oeste de Nicaragua IDR-GTZ.

Sabogal, C; Valerio, L. 1998. Forest composition, structure and regeneration in a dry forest of the Nicaraguan Pacific coast. In: Forest Biodiversity in North Central and South America, and the Caribbean: Research and Monitoring. F Dallmeier and J.A. Comiskey (eds.). Man and The Biosphere Series, Vol. 21. UNESCO. New York, USA. p. 187-212.

Sáenz, GP; Finegan, B. 2000. Monitoreo de la regeneración natural con fines de manejo forestal. Manejo Forestal Tropical 15. CATIE. Turrialba, Costa Rica. Pp. 8.

Silver, WL; Ostertag, R; Lugo, AE. 2000. The potential for carbon sequestration through reforestation of abandoned tropical agricultural and pasture lands. Restor. Ecol. 8, 394-407.

Slocum, MG. 2000. Logs and fern patches as recruitment sites in a tropical pasture. Restor. Ecol. 8, 408413. 
Thomlinson, JR; Serrano, MI; del M. López, T, Aide, TM; Zimmerman, JK. 1996. Land-use dynamics in a post-agriculture Puerto Rican landscape (1936 - 1988). Biotropica. 28, 525-536.

Tucker, J; Brondizzio, ES; Moran, EF. 1998. Rates of forest regrowth in Eastern Amazonia: a comparison of Altamira and Bragantina regions, Para State, Brazil. Interciencia. 23, 1-10.

Wijdeven, SMJ; Kuzee, ME. 2000. Seed availability as a limiting factor in forest recovery processes in Costa Rica. Restor. Ecol. 8, 414-424.

Withmore, TC. 1998. A pantropical perspective on the ecology that underpins management of tropical secondary rain forest. In: Ecology and Management of tropical secondary forest: Science, People, and Policy. Serie Técnica, Reuniones Técnicas N 4. CATIE, Turrialba, Costa Rica, pp. $19-34$.

Zimmerman, JK; Pascarella, JB; Aide, TM. 2000. Barriers to forest regeneration in an abandoned pasture in Puerto Rico. Restor. Ecol. 8, 350-360. 